\title{
CORRECTION
}

Check for updates

Cite this: Chem. Commun., 2021 57,8520

DOI: $10.1039 / \mathrm{d} 1 \mathrm{cc} 90289$

rsc.li/chemcomm

\section{Correction: Hexagonal boron nitride nanosheet as an effective nanoquencher for the fluorescence detection of microRNA}

\author{
Xinyi Li, ${ }^{a}$ Song Chen, ${ }^{* a}$ Qian Liu, ${ }^{b}$ Yonglan Luo ${ }^{c}$ and Xuping Sun ${ }^{b}$
}

Correction for 'Hexagonal boron nitride nanosheet as an effective nanoquencher for the fluorescence detection of microRNA' by Xinyi Li et al., Chem. Commun., 2021, DOI: 10.1039/d1cc03011f.

The authors regret that the graph in Fig. 3B and the graphical abstract was missing curves $\mathrm{c}$ and $\mathrm{d}$. The graphical abstract has been updated and the corrected Fig. 3 is given below.
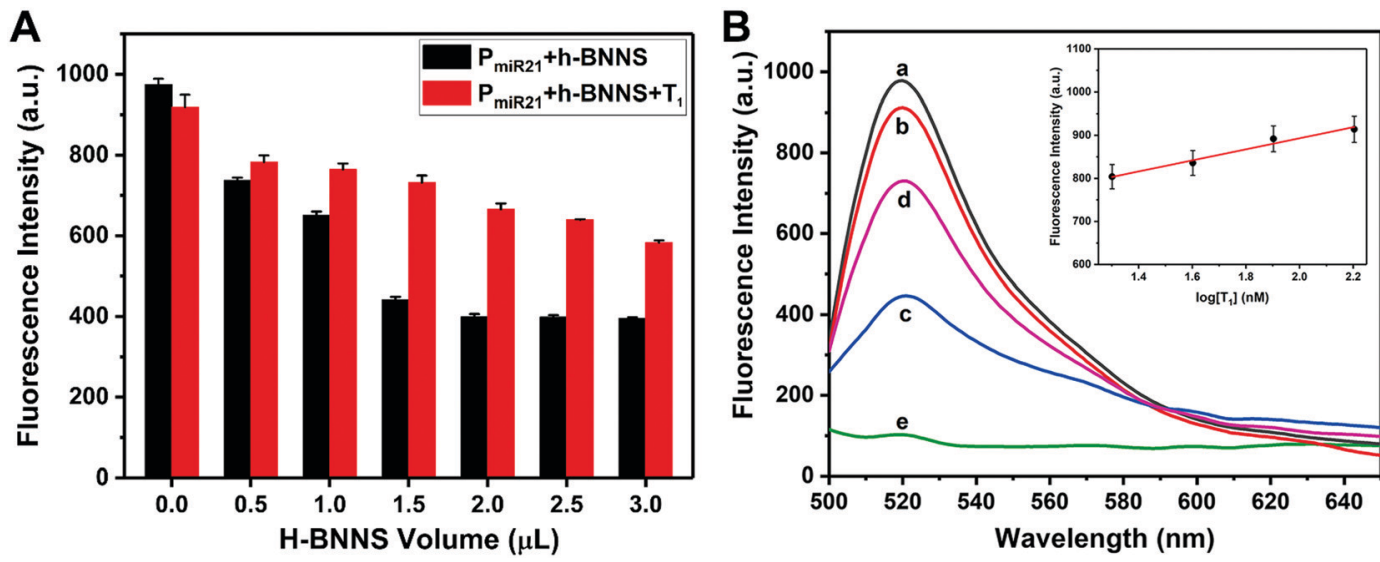

Fig. 3 (A) Fluorescence intensity histograms of $P_{\text {miR21 }}+h$-BNNS and $P_{m i R 21}+h$-BNNS $+T_{1}$ in the presence of $0,0.5,1.0,1.5,2.0,2.5$ and $3.0 \mu L$ of $h$-BNNS. (B) Fluorescence emission spectra of $P_{\text {miR21 }}$ under different conditions: (a) $P_{\text {miR21; }}$ (b) $P_{\text {miR21 }}+T_{1}$; (c) $P_{m i R 21}+h-B N N S$; (d) $P_{m i R 21}+h-B N N S+T_{1}$; and (e) h-BNNS. Inset: The fluorescence intensity of $P_{\text {miR21 }}+h$-BNNS plotted against the logarithm of the concentration of $T_{1}$.

The Royal Society of Chemistry apologises for these errors and any consequent inconvenience to authors and readers.

\footnotetext{
${ }^{a}$ State Key Laboratory of Oral Diseases, National Clinical Research Center for Oral Diseases, Department of Orthodontics, West China Hospital of Stomatology, Sichuan University, Chengdu 610041, Sichuan, China. E-mail: songchen882002@hotmail.com

${ }^{b}$ Institute of Fundamental and Frontier Sciences, University of Electronic Science and Technology of China, Chengdu 610054, Sichuan, China. E-mail: xpsun@uestc.edu.cn

${ }^{c}$ Institute for Advanced Study, Chengdu University, Chengdu 610106, Sichuan, China
} 